\title{
Monster Lung Cavity in a Heart Transplant Recipient
}

\author{
Brandon T. Nokes, MD, ${ }^{1}$ Octavio E. Pajaro, $\mathrm{MD}, \mathrm{PhD},{ }^{2}$ Jenise Stephen, PharmD, BCPS, ${ }^{3}$ \\ Patrick A. DeValeria, MD, ${ }^{2}$ Robert L. Scott, MD, PhD ${ }^{4}$ Sadia Z. Shah, MD, ${ }^{5,6}$ \\ D. Eric Steidley, $\mathrm{MD},{ }^{4}$ Jama Jahanyar, $\mathrm{MD}, \mathrm{PhD}^{2}$
}

\begin{abstract}
${ }^{1}$ Department of Internal Medicine, Mayo Clinic, Scottsdale, Arizona; and Departments of ${ }^{2}$ Cardiovascular and Thoracic Surgery, ${ }^{3} \mathrm{Pharmacy},{ }^{4}$ Cardiovascular Diseases, ${ }^{5} \mathrm{Pulmonary}$ Medicine and ${ }^{6} \mathrm{Critical}$ Care Medicine, Mayo Clinic Hospital, Phoenix, Arizona
\end{abstract}

\section{ABSTRACT}

Invasive mucormycosis infections occur in less than $1 \%$ of recipients of orthotopic heart transplants. Given the angioinvasive nature of these infections, the mortality rate is high. Little literature exists regarding the presentation and management of these infections. We present a case of a patient who developed an infection after orthotopic heart transplant, describe the successful multidisciplinary management surrounding his care, and review the available literature regarding mucormycosis infections in heart transplant recipients.

\section{CASE REPORT}

Invasive mucormycosis infections occur in less than $1 \%$ of recipients of orthotopic heart transplants, although this number is increasing [Petrikkos 2012; Abidi 2014]. Given the angioinvasive nature of these infections, the mortality rate is high [Tan 1999; Bhagat 2016]. Here we present an unusual case of pulmonary mucormycosis complicated by a rapidly evolving, massive cavitation. Our patient's infection was successfully managed by prompt surgical resection and longterm antifungal therapy.

A 66-year-old man came to our Emergency Department after having chills, night sweats, and hemoptysis (approximately "2 tablespoons," 10 times per day) for 2 days. He had undergone an orthotopic heart transplant 1.5 years earlier for ischemic cardiomyopathy and class IIIB congestive heart failure. His posttransplant history was uncomplicated except for an episode of grade 2 rejection (International Society for Heart \& Lung Transplantation criteria). At time of transplant, he received induction therapy with antithymocyte globulin, after which he was given a maintenance immunosuppressive regimen of tacrolimus, mycophenolate mofetil, and prednisone for 10 months. He was also taking prophylaxis for opportunistic infections with sulfamethoxazole/trimethoprim, fluconazole, and valganciclovir for the recommended time periods. His medical history before transplant also included hypertension; moderately well-controlled,

Received October 25, 2017; accepted February 1, 2018.

Correspondence: Jama Fabanyar, MD, PhD, Department of Cardiovascular and Thoracic Surgery, Mayo Clinic Hospital, 5777 E Mayo Blvd, Phoenix, AZ 85054; 480-342-2270 (email: jahanyarjama@mayo.edu). insulin-dependent diabetes mellitus (hemoglobin $[\mathrm{Hb}] \mathrm{A}_{1 \mathrm{c}}$ of 7.3), gout; obesity, and sleep apnea.

A chest radiograph and then a computed tomography scan showed a cavitary lesion of the left lower lobe (Figure 1A-D), suggestive of invasive fungal infection. Medical therapy (amphotericin B, posaconazole, and caspofungin) was initiated for the presumed infection. Bronchoscopy was performed, and purulent secretions were found in the left lower lobe. Lavage and transbronchial biopsies were done and cultures obtained, which did not show a specific bacterial organism. However, the patient's Aspergillus antigen level was somewhat increased. Concurrent serologic testing for coccidioidomycosis by enzyme immunoassay, complement fixation, and immunodiffusion was negative. Despite 8 days of conservative treatment, the cavitary lesion continued to expand on follow-up computed tomography, prompting the patient's immediate referral to surgery.

The patient underwent an urgent, open left lower lobectomy (Figure 2) and was treated aggressively with antifungal therapy. The lobectomy was complicated because the cavity was densely attached to the parietal pleura, but the pleura was not contaminated. To avoid intrapleural spillage, the pleura was left attached to the cavity for the lobectomy. Cultures taken during surgery were positive for Rbizopus (Zygomyces species), and pulmonary mucormycosis with necrotizing pneumonia was the final diagnosis. Caspofungin was discontinued, as Zygomyces is resistant to echinocandins, but the patient continued to take posaconazole and amphotericin B. Posaconazole was subsequently transitioned to isavuconazole sulfate, and amphotericin B was continued twice per week, as tolerated. Eventually, the patient took only isavuconazole for maintenance therapy. Follow-up imaging 1 year later did not reveal any evidence of recurrence (Figure 3 ).

\section{DISCUSSION}

Mortality rate for patients with pulmonary mucormycosis is high, given the angioinvasive nature of these infections. Reported risk factors are most often long-term immunosuppression (especially in patients with neutropenia), diabetes mellitus, and stem cell transplant [Williams 2014], as well as prior voriconazole or caspofungin use [Schowengerdt 1997; Grossi 2000; Ko 2000; Petrikkos 2012], although voriconazole exposure as an independent risk factor has been refuted [Abidi 2014]. Rarely, fungal coinfections have been reported 

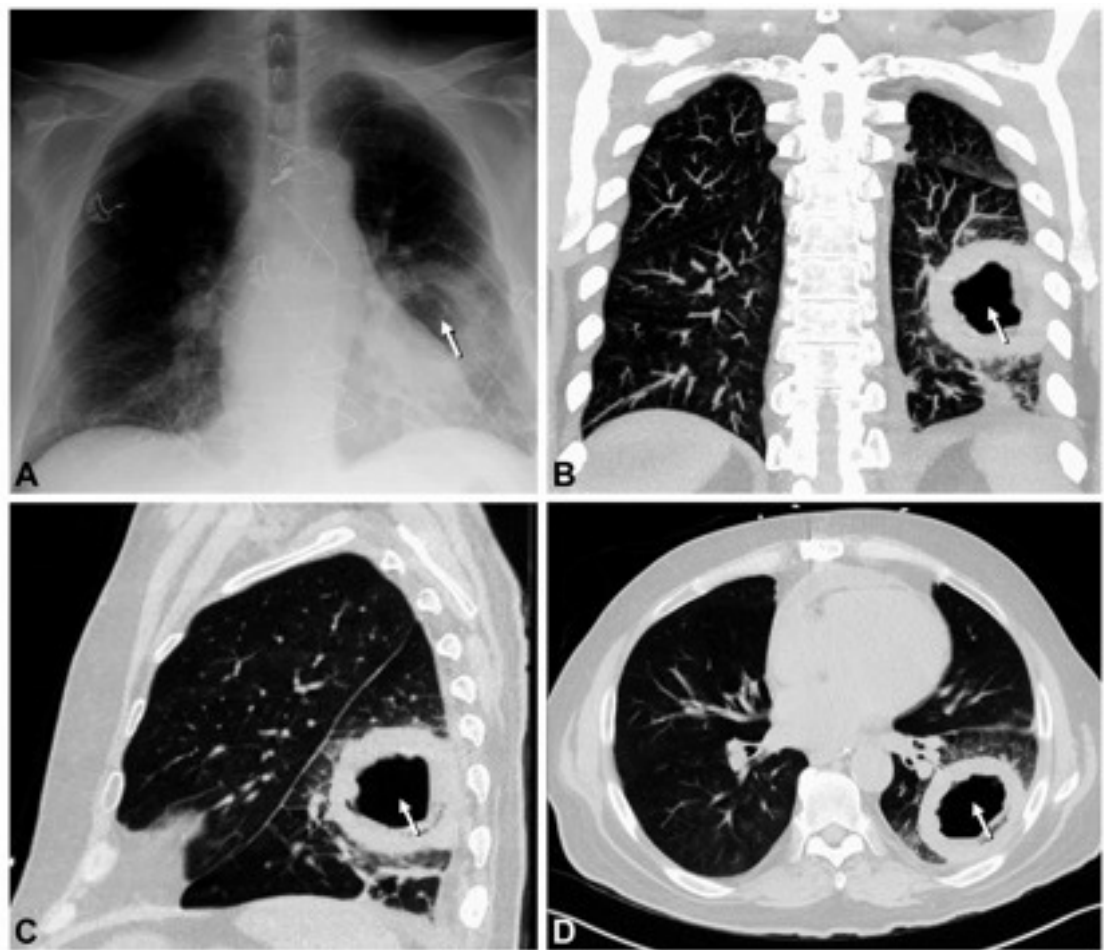

Figure 1. A, Chest radiograph. B-D, computed tomography scan of the chest revealing a large cavitary left lower lobe lesion with surrounding necrotic lung tissue.
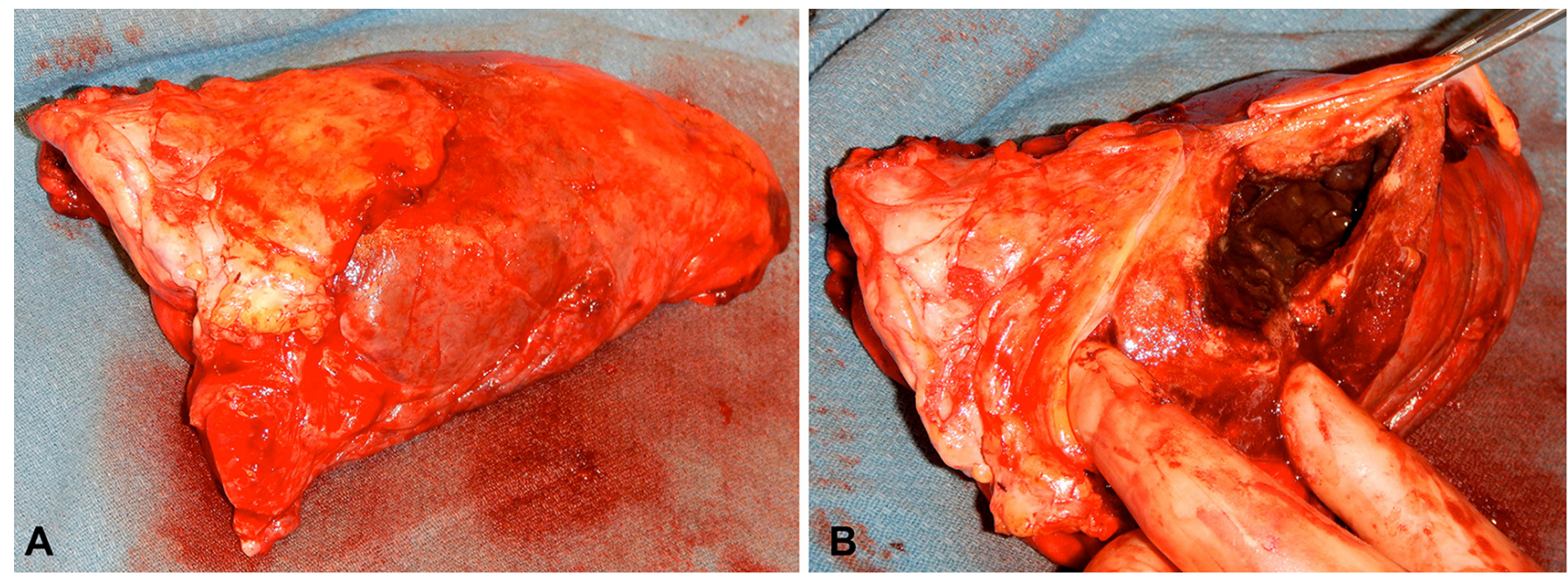

Figure 2. A and B, Gross section of left lower lobectomy revealing a large, cavitary lesion.

in heart transplant recipients [Webb 2013]. The immunosuppressant tacrolimus has been associated with an overall decreased risk of mucormycosis in solid organ transplant for reasons that are unclear [Schowengerdt 1997; Grossi 2000; Ko 2000; Petrikkos 2012].

Rhizopus is the most common fungal genus associated with mucormycosis. The fungus can infect various parts of the body, including the lungs, cutaneous tissues, gastrointestinal tract, sinuses, nasal passages, oral cavity, and brain (rhinocerebral), and, rarely, kidneys [Petrikkos 2012]. The fungal infection can be disseminated and can also occasionally cause endocarditis, peritonitis, and osteomyelitis [Petrikkos 2012]. Under normal circumstances, cellular immunity prevents the propagation of invasive Rhizopus infections. However, in immunosuppressed persons, these infections can rapidly expand into the pulmonary vasculature, causing frank 


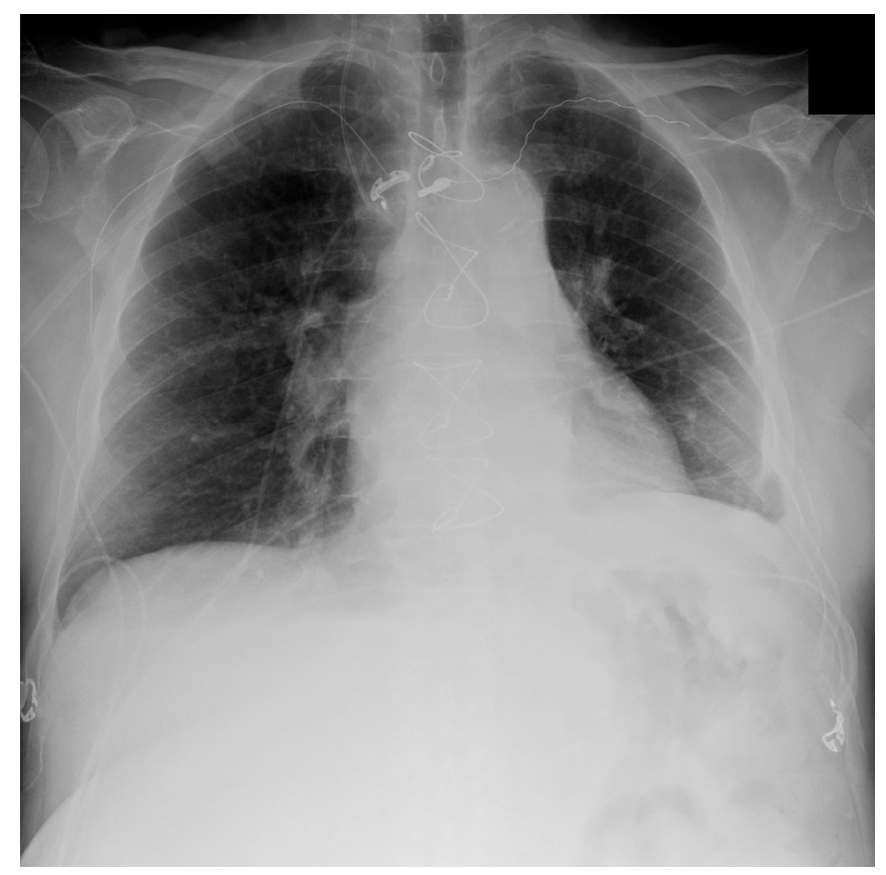

Figure 3. Chest radiograph at 1-year follow-up showing interval resolution of the prior cavitary lesion following left lower lobectomy.

hemoptysis and cardiopulmonary collapse. The appearance of a cavity imparts greater risk of hemoptysis. On radiography, mucormycosis infections typically appear as lobar consolidations, isolated masses, nodular disease, and, least commonly, cavitation or wedge-shaped infarctions [Spellberg 2005].

The mainstay of management in these cases is prompt surgical resection. Heretofore, these types of fungal infections were treated predominantly with amphotericin B. However, newer antifungal agents (e.g., isavuconazole) have broadened options for systemic therapy. No added benefit is gained from dual antifungal therapy or from adding the iron chelator deferasirox [Spellberg 2005; Spellberg 2009; Abidi 2014].

\section{CONCLUSION}

In conclusion, our patient survived because of our early and aggressive surgical intervention and care management from a multidisciplinary team, including cardiothoracic surgery, cardiology, infectious disease, pulmonology, and transplant pharmacy. Over the following year, the patient was able to return to his functional baseline without any pulmonary compromise, evidence of recurrence, or subsequent hospitalizations.

\section{REFERENCES}

Abidi MZ, Sohail MR, Cummins N, et al. 2014. Stability in the cumulative incidence, severity and mortality of 101 cases of invasive mucormycosis in high-risk patients from 1995 to 2011: a comparison of eras immediately before and after the availability of voriconazole and echinocandin-amphotericin combination therapies. Mycoses 57:687-698.

Bhagat M, Rapose A. 2016. Rapidly progressing dual infection with Aspergillus and Rhizopus: when soil inhabitants become deadly invaders. BMJ Case Rep Dec 8;2016.

Grossi P, Farina C, Fiocchi R, Dalla Gasperina D. 2000. Prevalence and outcome of invasive fungal infections in 1,963 thoracic organ transplant recipients: a multicenter retrospective study. Italian Study Group of Fungal Infections in Thoracic Organ Transplant Recipients. Transplantation 70:112-116.

Ko WJ, Chien NC, Chou NK, et al. 2000. Infection in heart transplant recipients: seven years' experience at the National Taiwan University Hospital. Transplant Proc 32:2392-2395.

Petrikkos G, Skiada A, Lortholary O, et al. 2012. Epidemiology and clinical manifestations of mucormycosis. Clin Infect Dis 54 Suppl1:S23-34.

Schowengerdt KO, Naftel DC, Seib PM, et al. 1997. Infection after pediatric heart transplantation: results of a multiinstitutional study. The Pediatric Heart Transplant Study Group. J Heart Lung Transplant 16:1207-1216.

Spellberg B, Edwards J, Jr., Ibrahim A. 2005. Novel perspectives on mucormycosis: pathophysiology, presentation, and management. Clin Microbiol Rev 18:556-569.

Spellberg B, Walsh TJ, Kontoyiannis DP, Edwards J, Jr., Ibrahim AS. 2009. Recent advances in the management of mucormycosis: from bench to bedside. Clin Infect Dis 48:1743-1751.

Tan HP, Razzouk A, Gundry SR, Bailey L. 1999. Pulmonary Rhizopus rhizopodiformis cavitary abscess in a cardiac allograft recipient. J Cardiovasc Surg (Torino) 40:223-226.

Webb BJ, Blair JE, Kusne S, et al. 2013. Concurrent pulmonary Aspergillus fumigatus and mucor infection in a cardiac transplant recipient: a case report. Transplant Proc 45:792-797.

Williams KE, Parish JM, Lyng PJ, et al. 2014. Pseudomembranous tracheobronchitis caused by Rhizopus sp. After allogeneic stem cell transplantation. J Bronchology Interv Pulmonol 21:166-169. 